\title{
Optimization of Yeast Protein Extraction through a Combined Enzymatic and High-Pressure Homogenization Method ${ }^{+}$
}

\author{
Otilia-Gabriela Bărbieru 1,2, Naomi Tritean 1,3, Bogdan Trică ${ }^{1}$, Diana Constantinescu-Aruxandei 1 \\ and Florin Oancea ${ }^{1, *}$ \\ 1 National Institute for Research \& Development in Chemistry and Petrochemistry - ICECHIM, \\ 060021 Bucharest, Romania; otilia-gabriela.barbieru@icechim.ro (O.-G.B.); naomi.tritean@icechim.ro (N.T.); \\ bogdan.trica@icechim.ro (B.T.); diana.constantinescu@icechim.ro (D.C.-A.) \\ 2 Faculty of Biotechnologies, University of Agronomic Sciences and Veterinary Medicine of Bucharest, \\ Bulevardul Mărăști, 59, 011464 Bucharest, Romania \\ 3 Faculty of Biology, University of Bucharest, Splaiul Independentei 91-95, 050095 Bucharest, Romania \\ * Correspondence: florin.oancea@icechim.ro \\ † Presented at the 16th International Symposium "Priorities of Chemistry for a Sustainable Development" \\ PRIOCHEM, Bucharest, Romania, 28-30 October 2020.
}

Published: 13 November 2020

Keywords: yeast extract; optimization; response surface methodology

Yeasts have a very high content of protein; therefore, they could be used as an easy source of protein. Spent brewer's yeast is a good substrate to use, as it is produced in large quantities from the brewing industry and it offers a great way of utilizing a subproduct to create value-added products, such as plant biostimulants [1].

Protein hydrolysates have been used as plant biostimulants for a long time and they have been proved to be efficient in mitigating the side-effects of harsh climatic conditions, such as droughts and cold weather [2]. The aim of this study is to present an optimization method for the extraction of yeast proteins, through enzymatic treatment coupled with high pressure homogenization. The protein extraction from yeast was carried out following several steps. The yeast was pre-treated with a $\beta$-glucanase at different concentrations, for one hour at $50{ }^{\circ} \mathrm{C}$ and afterwards lysed through a homogenizer at different pressure levels and different number of passes. The experiments were planned out through response surface methodology, based on a plan of two levels and four variable factors, which were temperature, pressure, number of passes and $\beta$-glucanase concentration. Pressure and $\beta$-glucanase concentration were highly significant $(p<0.05)$, while yeast concentration was only marginally significant when it comes to establishing a model. The interaction between two factors was also taken into consideration and the data show that the interaction between yeast-enzyme concentrations-yeast concentration-pressure were not significant. However, the interaction between enzyme concentration and pressure was marginally significant. The data analysis provided a set of optimal values for the predefined parameters in order to create a better process with a higher yield of proteins from yeast which could be used for other applications.

Acknowledgments: This work was funded by Subsidiary contract 1519/2019 of project POC-A1-A1.2.3-G-2015-P_40-352- "Sequential processes of closing the side streams from bioeconomy and innovative (bio)products resulting from it"(SECVENT) 81/2016 funded by cohesion funds of the European Union. 


\section{References}

1. Heuzé, V.; Thiollet, H.; Tran, G.; Edouard, N.; Lessire, M.; Lebas, F. Brewers Yeast. Feedipedia, a Programme by INRA, CIRAD, AFZ and FAO. 2018. Available online: https://feedipedia.org/node/72 (accessed on 1 October 2020).

2. Colla, G.; Rouphael, Y.; Canaguier, R.; Svecova, E.; Cardarelli, M. Biostimulant action of a plant-derived protein hydrolysate produced through enzymatic hydrolysis. Front. Plant Sci. 2014, 5, 448.

Publisher's Note: MDPI stays neutral with regard to jurisdictional claims in published maps and institutional affiliations.

(C) 2020 by the authors. Licensee MDPI, Basel, Switzerland. This article is an open access article distributed under the terms and conditions of the Creative Commons Attribution (CC BY) license (http://creativecommons.org/licenses/by/4.0/). 